\title{
L'appropriation du modèle du Louvre par les musées de province au tournant du XIX siècle
}

The appropriation of the model of the Louvre by the provincial museums at the turn of the nineteenth century

\section{Camille Doutremépuich}

\section{(2) OpenEdition}

1 Journals

Édition électronique

URL : http://journals.openedition.org/cel/794

DOI : $10.4000 /$ cel.794

ISSN : 2262-208X

Éditeur

École du Louvre

Référence électronique

Camille Doutremépuich, «L'appropriation du modèle du Louvre par les musées de province au tournant du xix e siècle », Les Cahiers de l'École du Louvre [En ligne], 11 | 2017, mis en ligne le 26 octobre 2017, consulté le 17 septembre 2019. URL : http://journals.openedition.org/cel/794 ; DOI : 10.4000/ cel.794

Ce document a été généré automatiquement le 17 septembre 2019.

\section{c) (7) $(9$}

Les Cahiers de l'École du Louvre sont mis à disposition selon les termes de la licence Creative Commons Attribution - Pas d'Utilisation Commerciale - Pas de Modification 4.0 International. 


\title{
L'appropriation du modèle $d u$ Louvre par les musées de province au tournant du XIX ${ }^{\mathrm{e}}$ siècle
}

\author{
The appropriation of the model of the Louvre by the provincial museums at the \\ turn of the nineteenth century
}

Camille Doutremépuich

1 Lorsque l'on aborde la question du modèle du Louvre, il est relativement légitime de le considérer comme un modèle universel. Le Louvre, depuis sa création, tend à se revendiquer comme tel, et le rayonnement international dont il bénéficie renforce sa dimension universaliste. Si le Louvre et son modèle constituent un exemple, ou en tout cas un point convergent d'intérêts multiples, nationaux et internationaux, ce grand musée à vocation encyclopédique s'insère néanmoins dans une histoire complexe des musées de France, dont les nuances incitent à se pencher sur les différents degrés et facettes de la construction de ces institutions.

2 L'intérêt que ces musées ont suscité n'a été que croissant à partir de la Révolution, de nombreux auteurs se sont ainsi attelés à mettre en lumière les étapes et les particularités de cette histoire, nous léguant, dès le XIX ${ }^{e}$ siècle, un travail précieux ${ }^{1}$. La promotion de ces institutions par l'inspecteur des musées de province Philippe de Chennevières, qui publia de nombreux essais sur la question entre 1847 et $1865^{2}$, est un formidable témoignage des moyens mis en œuvre (avec parfois beaucoup de difficultés) pour pérenniser l'existence des musées. L'ouvrage de Clément de Ris, Les musées de province: histoire et description ${ }^{3}$, pose quant à lui les jalons d'une histoire des musées de province, et soulève déjà des questionnements autour du modèle du Louvre, à travers l'élaboration des catalogues de musée :

Serait-ce se montrer bien exigeant que d'imposer aux conservateurs chargés de la rédaction des catalogues, l'obligation de prendre pour modèle un type unique; le catalogue du Louvre? Ne contribueraient-ils pas ainsi, chacun pour leur part et sans grands efforts à la rédaction d'un inventaire complet des œuvres d'art 
disséminées sur le sol de la France? Et ne serait-ce pas là un des plus beaux monuments élevés à l'histoire ${ }^{4}$ ?

Pour Philippe de Chennevières et Clément de Ris, les musées de province fournissent l'occasion de dresser un catalogue complet de l'art et de l'histoire de France, notamment à travers les œuvres conservées au sein des musées - puisque, selon les mots de Chennevières, il s'agit bien d'un fonds d' "art provincial» auquel on ne demande que de représenter "le génie de sa province ${ }^{5}$ - en se fondant essentiellement sur le nouveau modèle de catalogage du Louvre établi en partie par Frédéric Villot, et publié dès $1849^{6}$. Philippe de Chennevières salue néanmoins le travail effectué dans le catalogue du musée de Valenciennes ${ }^{7}$, pourtant antérieur au catalogue de Villot, dont les notices biographiques sur les artistes, et notamment sur les artistes locaux oubliés ou dont les œuvres ont été détruites, ont été richement développées ${ }^{8}$. Ces initiatives successives sont le fruit d'une demande constante du public de catalogues plus détaillés (Stéphane Loire nous fait remarquer une montée des critiques dans les années $\left.1830-1840^{\circ}\right)$. Si au Louvre le catalogue devient l'objet d'un véritable travail scientifique, dont le modèle sera diffusé en France et en Europe, nous l'avons vu, ce dernier doit toutefois être adapté aux destinations des musées de province.

Cette question nous amène finalement à nous interroger sur ces "destinations » des musées de province. Sont-ils perçus comme des succursales du Louvre? Ou, au contraire, comme des entités ayant pour nécessité d'affirmer une indépendance aussi bien scientifique que fonctionnelle? D'ailleurs, peut-on parler d'unité pour ces institutions? Et quelles sont les réalités des musées de province face à une forme d'idéalisation collective, ou encore face à l'opinion publique? Enfin, le Louvre a-t-il inspiré un modèle durable en France?

\section{Le musée, objet de débat}

Les nombreuses sources qui nourrissent le débat sur la place du musée témoignent de l'intérêt général porté à ces institutions récentes, attisant aussi bien la curiosité des administrateurs, des critiques, des artistes que du public. Cette question fait l'objet de discussions, parfois passionnées, essentiellement à partir de la période révolutionnaire $^{10}$. Le peintre et marchand d'art Jean-Baptiste-Pierre Lebrun fait imprimer dès 1792 et 1793 ses Réflexions et Observations sur le Muséum national, alimentant le débat sur la constitution des musées (puisqu'il parle aussi des musées des départements) ${ }^{11}$. Le Muséum national doit annoncer «toute la grandeur du peuple français", et Paris doit recueillir "dans son sein tous les chefs-d'œuvre de la République $»^{12}$. Cette conception hégémonique du Muséum central est présente dans de nombreux discours, dont ceux des ministres successifs qui s'accordent sur le fait que Paris doit conserver les plus grands chefs-d'œuvre.

Lebrun propose en outre, un peu à contre-courant, de fonder les musées des départements dans des villes peu peuplées, où la production et le commerce ne sont pas développés, ajoutant qu'ils doivent rester en petit nombre. Il semble que Lebrun perçoive les musées de province comme des miniaturisations du Louvre, avec des œuvres de second plan. La pensée générale ne suit pas nécessairement cet avis tranché : Louis-Georges de Bréquigny, érudit et alors président de la Commission pour la création de dépôts, préfère l'établissement des musées, encore appelés « dépôts ", au sein de grandes villes, de préférence densément peuplées, où «il y aurait un 
établissement d'instruction publique $»^{13}$. Avec la récente nationalisation des biens de la couronne, du clergé et des émigrés, les administrateurs et hommes politiques cherchent à garantir le "progrès des arts " et leur "régénération ${ }^{14}$, en rendant accessibles les collections et en les étendant de surcroît à l'ensemble du territoire, pour le bien de l'instruction publique.

7 Les gouvernements successifs tentent de légiférer et de donner une méthode d'application pour ces institutions en devenir, dont l'organisation demeure instable. Les premiers décrets sont généralement établis en vue d'une répartition des œuvres sur le territoire, mais aussi pour préserver les trésors du Louvre qui ouvre ses portes le 10 août $1793^{15}$. Les œuvres sont ainsi classées par catégorie, les plus méritoires réservées au Louvre ou au nouveau musée historique de Versailles, le reste "pour la réserve, c'est-à-dire pour servir soit aux échanges, soit à la formation d'autres Musées ${ }^{16}$ ». La question de ponctionner les chefs-d'œuvre confisqués au sein des départements à destination du Muséum central est même débattue en septembre 1793, juste après l'ouverture du Louvre au public en août ${ }^{17}$; mais l'idée n'aboutit pas, puisqu'un décret jugeant que les œuvres seront conservées dans le «musée le plus voisin » du lieu où elles ont été saisies est publié ${ }^{18}$. Certaines villes de province protestent d'ailleurs avec vigueur contre cette volonté de concentration des œuvres et objets d'art, comme à Rennes où une pétition est signée pour l'ouverture d'un musée, ou encore à Rouen, Dijon ou Angers, ce qu'Édouard Pommier relève avec soin dans un long article sur la "Naissance des musées de province ${ }^{19}$ ". On constate à travers ces différents événements et démarches la volonté de promouvoir l'égalité entre Paris et la province, conduisant au développement d'une politique culturelle solide, et une volonté d'autonomie et de reconnaissance au sein des régions.

8 Vient, quelques années plus tard, le fameux arrêté consulaire du 14 fructidor an IX ${ }^{20}$, dit aussi "arrêté Chaptal », qui constituerait l'acte de naissance des musées de France. Ce texte, sans nul doute fondateur, donne davantage un crédit administratif à quinze musées $^{21}$, en les officialisant, qu'il ne permet leur création effective, qui, par ailleurs, pourrait sembler soudaine. Édouard Pommier ${ }^{22}$ revient très clairement sur la manière dont doit être envisagée cette histoire. Une histoire qui n'est linéaire ni dans le temps, ni dans l'espace; c'est à tort que l'on chercherait un point d'origine de l'existence des musées, qui est plutôt la manifestation d'un phénomène général autour d'une revendication culturelle, et - Édouard Pommier insiste sur ce point - qu'elle soit locale ou nationale : «Il pourrait sembler [...] artificiel de disjoindre le cas de la province de celui de la capitale, ou, autrement dit, le phénomène local du phénomène national ${ }^{23}$. " La période révolutionnaire et celle qui suit sont le moment d'une "construction mythique [...] d'une histoire raisonnable et morale ", selon les termes de Dominique Poulot $^{24}$, à travers la constitution d'un patrimoine, et dont le vecteur principal s'incarne dans la figure du musée.

$9 \quad$ L'existence des musées passe d'abord par l'appropriation des œuvres qui tendent à être considérées comme appartenant au bien commun - ce que la période révolutionnaire contribue à créditer, en désignant ce patrimoine annexé comme étant inaliénable et devant profiter à tous les citoyens. Quelques exemples de cette vocation à rendre publiques des collections privées peuvent néanmoins être remarqués très tôt sur le territoire français. L'exemple, souvent considéré comme la plus ancienne forme de collection «publique » en France, serait celui du legs Boisot à Besançon. L'abbé JeanBaptiste Boisot légua sa collection en 1694 aux bénédictins de Saint-Vincent, « à charge 
et condition que tout sera mis dans une salle qui sera ouverte deux fois la semaine à tous ceux qui voudront y entrer, lesquels pourront lire et étudier autant qu'ils le souhaitent ${ }^{25}$ ». Il créa en quelque sorte, sans le savoir, le premier «musée » de France, bénéficiant d'un contrôle municipal et d'une certaine publicité en ville, jusqu'en 1790 où la collection fut intégrée dans les saisies révolutionnaires. D'autres cas de collections rendues publiques, qu'elles soient prises en charge par des municipalités ou des sociétés savantes, sont connus tout au long du XVIII ${ }^{e}$ siècle $^{26}$.

10 Si la revendication d'un accès aux œuvres d'art culmine à la Révolution, l'idée de construire un espace public pour accueillir les collections est déjà présente au XVIII ${ }^{\mathrm{e}}$ siècle, aussi bien en province à travers les exemples évoqués, qu'au Louvre où l'on commence à aménager les espaces d'exposition et d'étude pour les artistes, sans compter la tenue du Salon à un rythme bisannuel dès 1751. La multiplication des initiatives, parfois indépendantes dans les années 1780 - La Rochelle (1782), Bordeaux (1783), Arles (1784), Nîmes (1785), Dijon (1787), etc. - montre que la création du musée est devenue un enjeu non seulement politique, mais aussi social et économique. Le musée, à l'origine lieu d'enseignement, d'apprentissage, aussi bien pour les artistes que pour le public, qui peut désormais avoir accès à une forme d'éducation, devient un objet central du développement de la vie culturelle au sein des villes.

\section{L'enjeu identitaire des musées}

11 Le Louvre, à travers son projet universel et encyclopédique, devenu possible grâce à la réunion des œuvres issues des saisies révolutionnaires et européennes, se veut être l' exemplum virtutis, conforme à des valeurs patrimoniales mais aussi patriotiques. Le caractère national et universaliste du Louvre - qui détient des chefs-d'œuvre de toutes les écoles, des objets d'arts de plusieurs civilisations - montre l'enjeu d'une représentation du musée français, de la construction de l'identité de la Nation. Quant aux musées de province, initialement conçus en lien étroit avec les écoles de dessin déjà en place ${ }^{27}$, et dans un premier temps presque exclusivement à destination des artistes en apprentissage (les directeurs des écoles de dessin cumulent souvent leur poste avec celui de conservateur du musée, et ce sont eux qui, la plupart du temps, ont inventorié les biens saisis lors de la Révolution: François Devosge et son fils Anatole à Dijon, Charles-Antoine Rougeot et son gendre Jean-Jacques Raverot à Tours, Pierre Lacour à Bordeaux, Pascal-Jean Lenot à Mâcon...), ils développent eux aussi une conceptualisation de l'objet-musée, en tant que lieu identitaire propre à la valorisation d'un patrimoine, généralement local.

On constate une progression simultanée dans l'évolution de la constitution des collections au sein des musées de province. D'un côté, le modèle universel du Muséum incarné par le Louvre, dont le but encyclopédique tend à pourvoir l'institution d'une grande diversité d'objets et d'œuvres d'art - en somme, établir un panorama - comme le concevait déjà le comte d'Angiviller. Le Louvre canonise ainsi la tradition muséale, et pérennise l'idée du temple des arts et de l'histoire qui se doit d'honorer la Nation. Cette volonté encyclopédique se retrouve en partie au sein des musées de province, qui doivent réunir les modèles nécessaires à l'instruction des élèves se destinant aussi bien aux arts dits libéraux que mécaniques. Le ministre de l'Intérieur Pierre Bénézech avait d'ailleurs fait part de son intention de voir chaque musée contenir essentiellement « des objets analogues au but de son établissement ${ }^{28}$ ». Les envois de l'État caractérisent 
particulièrement cet aspect généraliste des collections au sein des musées ; les premiers envois, dont celui de 1801, comptent des œuvres des grands maitres, incontournables à l'étude pratique et à l'édification des connaissances.

Parallèlement, on retrouve au sein des collections un second noyau, illustrant la volonté de favoriser les arts du pays, de promouvoir les artistes locaux qui se sont illustrés ou, au contraire, sont tombés dans l'oubli (voir le catalogue du musée de Valenciennes évoqué précédemment), mais aussi des grandes figures qui ont œuvré pour la région - on pense notamment à Toulouse où le musée, créé dès 1795, expose, à l'initiative de Jean Briant, les tableaux historiques conservés dans les collections du Capitole, dont les portraits des Capitouls ${ }^{29}$. Plus étonnant encore, Briant organise au début de l'année 1796 une petite exposition de quinze œuvres d'artistes du pays toulousain ${ }^{30}$.

14 Les collections des musées de province s'organisent ainsi fréquemment selon deux axes. L'un est formé par les œuvres, dessins, gravures et plâtres réunis par et pour les écoles de dessin ainsi que par les envois de l'État, dont la nature est plutôt généraliste, ou qui du moins présente un panorama de la peinture ; l'autre est constitué d'œuvres plus ancrées dans l'histoire locale, œuvres saisies lors de la Révolution, réalisées par des élèves de l'école de la ville, ou encore issues d'un legs ou d'un don - pratique qui caractérise de nombreuses collections en France, et qui a modifié l'essence même des musées, en modelant parfois leur image de manière durable, comme à Lille avec la collection Wicar ${ }^{31}$.

Nous sommes loin de la conception des musées de province comme succursales du Louvre, comme pouvait l'évoquer le marchand et peintre Lebrun, et il est de surcroît difficile d'envisager une unité absolue des musées français, dont l'origine est infiniment variée, et la genèse profondément inhérente à une histoire locale - les initiatives personnelles ou collectives (on pense notamment aux sociétés amicales qui ont parfois joué un rôle indéniable dans la construction et la pérennisation de certaines institutions) ont formé le paysage culturel des villes. On reconnaît différentes ambitions à travers les collections et la structuration de ces musées, tantôt à vocation universelle, tantôt à vocation commémorative, exaltant souvent une fierté locale.

La spécialisation croissante de ces musées, créant une grande diversité dans le champ de ces institutions, a permis de dessiner une cartographie des spécificités des histoires régionales - on peut citer les musées d'histoire comme celui de Toulouse, les musées archéologiques comme à Arles ou à Bordeaux, les musées consacrés à des personnages ou des artistes locaux, comme l'espace d'exposition dédié à David d'Angers dans le musée des Beaux-Arts d'Angers dès 1838, ou encore Dijon, avec l'aménagement entre 1823 et 1828 de la salle des Gardes pour accueillir les tombeaux des ducs de Bourgogne $^{32}$. Naturellement, les dons et les legs ont une place toute particulière dans les collections des musées, comme évoqués plus haut, au point que l'on parle parfois de ces institutions comme des collections de collections. Cette hétérogénéité qui les caractérise est donc le résultat d'une histoire multiple, qui se situe, comme l'a bien souligné Loïc Vadelorge, "au carrefour des différents espaces de légitimation culturelle ${ }^{33} »$. 


\section{Une appropriation... à double sens ?} musées, a pourtant une influence réelle sur la manière de structurer ces institutions. $\mathrm{Au}$ tournant $\mathrm{du}$ xIx $\mathrm{x}^{\mathrm{e}}$ siècle, l'attention se focalise sur les institutions parisiennes (le Louvre, le musée historique de Versailles, la Bibliothèque nationale, le musée des Monuments français), et de ce fait, les moyens d'œuvrer se concentrent eux aussi à Paris. Cependant, les gouvernements successifs encouragent les musées des départements à s'aligner sur les pratiques du Muséum central, concernant au moins l'organisation et la question des inventaires, avec la réalisation de catalogues des collections. Dominique Poulot souligne que, de 1793 à 1803, dix-sept catalogues sont publiés pour le Louvre, et neuf pour les Petits-Augustins ${ }^{34}$. Le musée de Toulouse publie le premier catalogue imprimé des musées de province en $1795^{35}$. La plupart des musées en province suivent cette démarche, dans un intérêt commun à tous. Les catalogues adoptent généralement le classement par école, comme celui du Muséum central, tout en devenant le moyen de valoriser les collections, comme nous l'avons vu au tout début à travers les propos de Philippe de Chennevières.

Mais le Louvre a-t-il pour autant déterminé les «normes» des musées de France? Comment les musées se sont-ils construits, aussi bien du point de vue de l'exposition des œuvres, de l'organisation générale que de l'architecture? Y a-t-il un modèle prédominant? les genres, juxtapose les chefs-d'œuvre, et défend ce choix en avançant qu'il s'agit d'un système "plus propre à développer le génie des élèves, et à former leur goût d'une manière sûre et rapide, en leur présentant sous un même point de vue des chefsd'œuvre en divers genres ${ }^{36}$ ». La raison officieuse est plutôt qu'il convient d'attendre que les collections soient complètement constituées et inventoriées.

Dès que l'inventaire est effectué, les collections sont classées par école, notamment pour « rappeler agréablement et sans peine l'histoire chronologique de l'art ${ }^{37}$ ». Les théorisations de la muséologie et de la gestion du musée sont débattues, plusieurs solutions sont mises en avant; Jean-Marie Roland de la Platière, ministre de l'Intérieur entre 1792 et 1793, était partisan d'un accrochage plus sensible, c'est-à-dire un agencement davantage coloré que didactique (qui fut appliqué par défaut en 1793). Le restaurateur Jean-Michel Picault, au contraire, avançait l'idée qu'en plus du classement par école, les œuvres devaient être d'une qualité homogène, restaurées et profiter d'un éclairage zénithal. Il semble que de nombreux musées aient adopté le classement chronologique et par école (qui prévaut encore de nos jours), à l'exception de quelquesuns, comme Toulouse que nous avons déjà évoqué, où Briant refuse le classement par école, préférant un musée historique, notamment à la gloire des grands hommes de la région. Concernant les préconisations sur l'éclairage et l'homogénéité des œuvres, il s'agissait là d'un défi plus difficile à relever pour l'ensemble des institutions, dont les collections souvent lacunaires ne bénéficiaient pas toujours des financements nécessaires à leur aménagement et à leur entretien. Elles devaient également faire face aux difficultés qui incombent à l'organisation d'un espace - hôtel de ville, église, halle qui ne se prête pas à l'exposition et à la bonne conservation des œuvres.

20 Le cas de Dijon est néanmoins exceptionnel de ce point de vue, puisque l'école de dessin et le musée disposèrent du soutien des États de Bourgogne, et prirent place au sein du 
palais qui était dédié à cette autorité régionale. Cela fut sans doute permis en grande partie grâce à l'investissement personnel et sans relâche de François Devosge, qui avait fondé l'école en 1766, et qui avait su convaincre la municipalité de l'intérêt du projet. Entre 1781 et 1786, l'aile orientale est adjointe au Palais des États de Bourgogne ${ }^{38}$. Cette aile orientale visait à accueillir l'école de dessin fondée par François Devosge, mais aussi à héberger la collection de plâtres et de dessins réunie par le directeur, qui s'enrichissait depuis quelque temps des envois de Rome des élèves de l'école - puisque François Devosge fut le seul directeur d'une école de dessin en province à avoir institué, dès 1770 , un concours quadriennal extraordinaire, qui récompensait les lauréats d'un séjour de quatre années à Rome.

Dès les années 1784-1786, la salle consacrée au musée - à un stade embryonnaire et davantage conçu comme un lieu d'enseignement - fait l'objet d'une grande commande de la part des États de Bourgogne afin de la décorer, et d'illustrer ainsi l'histoire de la Bourgogne à travers des épisodes glorieux de la vie du Grand Condé. Pierre-Paul Prud'hon envoie une œuvre à destination du plafond de la salle des Statues; tandis que Bénigne Gagneraux est chargé d'exécuter deux tableaux alors qu'il se trouve encore à Rome, qui prendront place dans la salle des Statues et dans le salon Condé, respectivement en 1788 et 1791.

Ces initiatives, qui peuvent nous paraître bien ordinaires, sont pourtant l'illustration d'une forme de conception du musée français. Édouard Pommier fait un constat des plus pertinents à ce propos : «À Dijon viennent confluer deux courants porteurs de l'idée de musée: l'Enseignement et la Mémoire; et l'institution s'établit dans la demeure du Pouvoir ${ }^{39}$." Sans affirmer que Dijon constitue le modèle initial, il s'agit simplement de remarquer les signes de l'appropriation d'un patrimoine et sa valorisation à travers l'exaltation d'une fierté locale, et cela dans un espace des plus chargés symboliquement : à la fois en sa qualité de lieu de pouvoir, et car le musée est vecteur de cette "régénération ${ }^{40}$ ", porteur des valeurs révolutionnaires et universalistes du progrès. Ce processus de patrimonialisation prend tout autant sens au Louvre, haut lieu du pouvoir monarchique. De manière générale, l'installation des musées dans les palais et les églises est, comme l'a dit Bruno Foucart, une manière de "sacraliser le contenu par le contenant ${ }^{41}$ ». D'ailleurs cette appropriation des lieux illustres est loin d'être une exception française : à Berlin, l'île aux musées, avec ses bâtiments aux allures de temples antiques, est toute proche du château royal, tandis qu'à Saint-Pétersbourg, le musée est le prolongement du Palais d'hiver des Tsars, dans la continuité que l'on retrouve à Paris avec le Louvre.

\section{L'aménagement des musées de province: une réalité précaire}

Si les musées prennent place en des lieux symboliques, religieux ou teintés de pouvoir, leur aménagement demeure très précaire. La Grande Galerie du Louvre put, certes, être rapidement réaménagée, mais les moyens mis en œuvre par les musées des départements étaient quant à eux nettement limités, et la problématique principale n'était pas d'arranger un espace trop spacieux, mais bien plus de s'accommoder de lieux exigus et impropres à la conservation d'œuvres d'art. Dès 1790, le rapport de Bréquigny devant la Commission des monuments interrogeait pourtant la manière $d$ ' abriter les dépôts. Il propose, de manière précoce, de réemployer les monuments 
désaffectés, et notamment les églises qu'il serait facile de réaménager en musées, en présentant une méthode de répartition des œuvres au sein des anciens édifices de culte ${ }^{42}$. Néanmoins cette belle intention ne suffisait pas à donner aux récents musées des conditions favorables à la conservation et à l'exposition des collections.

Les musées de Bordeaux, Marseille, Nancy, Nantes, Lille ou Rouen connaissaient alors de très mauvaises conditions de conservation. Le musée de Lille, logé dans l'étage supérieur de l'Hôtel de ville, décrit par Ludovic Vitet en ces termes : «[...] spacieux, mais assez mal éclairé, c'est une longue galerie où le jour ne pénètre que par deux grandes fenêtres, percées chacune à une extrémitée ${ }^{43}$ ", était propice aux incendies, et connut un vol en $1882^{44}$. Il fallut attendre 1892 pour qu'une grande souscription publique permette la construction d'un vaste palais des Beaux-Arts. Le musée de Bordeaux, qui connut de nombreux locaux depuis sa création, fut ravagé par deux incendies alors qu'il était installé au Palais Rohan, en 1862 et 1870. Il fut encore déplacé huit fois jusqu'en 1882, lorsque la municipalité fit enfin le nécessaire pour abriter le musée convenablement, en construisant deux grandes galeries au sein d'un jardin public $^{45}$.

Le bilan de l'inspection des musées de province de 1879-1880 montre d'ailleurs que les conditions dans lesquelles les musées sont logés sont encore très précaires. Dominique Poulot soulève ces chiffres : sur 82 musées inspectés, un quart bénéficie d'un bâtiment séparé (de la bibliothèque ou de l'école), la moitié est installée dans les hôtels de ville, et le reste se situe encore dans des logis divers ${ }^{46}$.

Les musées n'eurent pas le privilège de faire l'objet d'une construction adaptée dès leurs débuts. Les premières édifications débutèrent au deuxième quart $\mathrm{du} \mathrm{XIX}^{\mathrm{e}}$ siècle à Tours, où, un peu comme à Dijon, un bâtiment fut construit en 1825 pour accueillir le musée et l'école de dessin. Le musée de Besançon fut construit en 1844, celui de Grenoble en 1860. Entre 1815 et 1911, 42 musées sont construits, dont 31 sous la III République. Amiens constitue au demeurant un exemple intéressant de musée construit au milieu du xix ${ }^{e}$ siècle, souvent considéré comme une succursale du Louvre. Lors de la construction d'un bâtiment dédié au musée en 1852, Napoléon III, qui s'intéressa particulièrement au projet, céda un terrain, et c'est l'architecte Henri Parent qui se chargea de l'édification. Les salles furent définies pour classer les collections par école et de manière chronologique, avec un salon central pour les grands formats. Le plan et l'aménagement de la façade montrent que l'édifice a été conçu sur le modèle du Louvre de Napoléon III, proposé par Hector-Martin Lefuel et Louis Visconti. Le musée fut achevé en 1865, tandis qu'un envoi du Louvre gratifia le nouveau musée de 61 tableaux l'année précédente ${ }^{47}$. En dehors de cet exemple exceptionnel, qui intervient déjà tard dans le XIX ${ }^{\mathrm{e}}$ siècle, les musées eurent rarement le privilège de profiter de locaux adéquats ou construits spécifiquement pour eux au début du siècle.

\section{Conclusion}

Le Louvre est peut-être bien un modèle pour les musées de France, sans doute davantage après les années 1780-1800. Si le modèle du temple antique a prédominé en Europe et outre-Atlantique pour l'architecture muséale, le musée français se fonde quant à lui essentiellement sur le modèle du Palais - cela sans doute grâce à l'exemple du Louvre, qui «s'est imposé dans l'imaginaire national» selon Bruno Foucart ${ }^{48}$. L'exemple d'Amiens va en ce sens, et les exemples de Louvre miniatures se sont 
multipliés au milieu du XIX ${ }^{e}$ siècle, une fois le destin des musées relativement assuré. La possibilité de construire et d'aménager les espaces a permis, comme à Amiens, Rouen, Nantes ou Lille, de s'inspirer pleinement du modèle du Louvre, finalement considéré comme le musée à la française. Mais cette appropriation tardive du modèle du Louvre par les musées en région, essentiellement du point de vue architectural et muséologique, et les exemples précoces de collections rendues publiques dans toute la France, montrent bien que la province a joué un rôle, parfois capital, dans la conception du musée - notamment dans son orientation scientifique et dans le développement d'une politique d'exposition - et dans la genèse de son institutionnalisation.

\section{NOTES}

1. Voir Loïc Vadelorge, "Quelle histoire pour les musées de province? ", Les Musées de province dans leur environnement, actes de la journée d'étude, université de Rouen, 4 décembre 1993, sous la direction de Loïc Vadelorge, Mont-Saint-Aignan, Presses universitaires de Rouen, 1996, qui dresse une liste des descriptions classiques des musées de province dans sa première note (p.9).

2. Philippe de Chennevières publie de nombreux ouvrages au sujet de l'art en province: Travaux préparatoires et explicatifs [...] sur la nécessité de relier les musées des départements au musée central du Louvre, Paris, impr. de Lacour, 1848; Essais sur l'organisation des arts en province, Paris, J.B. Dumoulin, 1852; "Les musées de province », La Gazette des beaux-arts, $1^{\text {er }}$ février 1865, vol. XVIII, $\mathrm{n}^{\circ} 104$, pp. 118-131.

3. Clément de Ris, Les musées de province : histoire et description, 2 vols, Paris, J. Renouard, 1859-1861 (1859, éd. augmentée en 1872).

4. Idem, ibidem, vol. I, p. 11.

5. Philippe de Chennevières, «Les musées de province », op. cit. note 2, p. 122.

6. Pour une histoire détaillée des catalogues du Louvre de 1793 à nos jours, se référer à Stéphane Loire, " Les catalogues des peintures du musée du Louvre (1793-2013) », dans Le Catalogue dans tous ses états, collection "Rencontres de l'École du Louvre ", Cecilia Hurley Griener et Claire Barbillon (dir.), Paris, École du Louvre, 2015, pp. 171-194.

7. A. J. Potier, Livret historique des peintures, sculptures, dessins et estampes du musée de Valenciennes, Valenciennes, impr. de A. Prignet, 1841, p. 176 : « Notice sur les artistes valenciennois dont les ouvrages ont été détruits ou dispersés lors de la Révolution de 1793 ».

8. Philippe de Chennevières, «Les musées de province ", op. cit. note 2, p. 43 : « Pour achever mon plan de catalogue, je demanderais, citoyen directeur, que les peintres provinciaux ayant travaillé dans leur province, eussent, à la suite de l'indication de leurs travaux, une notice aussi complète que possible dont on resserrerait les faits dans une page. [...] Enfin le catalogue d'un Musée de province, pour être, comme il doit être en effet, le catéchisme d'art de sa province, réservera quelques pages (voyez pour modèle le catalogue de Valenciennes) où seront passés en revue et étudiés avec bienveillance les anciens peintres du pays dont le Musée n'a point de tableau mais dont le nom est resté fixé à quelque toile d'église, ou seulement dans un livre, ou seulement dans la mémoire populaire. »

9. Stéphane Loire, op. cit. note 6. 
10. Sur les débats autour de la formation du Muséum central, voir Dominique Poulot, «Le musée Souverain; Une attente déçue ", dans "Surveiller et s'instruire»: la Révolution française et l'intelligence de l'héritage historique, Oxford, Voltaire Foundation, 1996.

11. Jean-Baptiste-Pierre Lebrun, Réflexions sur le Muséum national, s.l., s.e., s.d. [1792]; Observations sur le Muséum national, Paris, chez Charon, 1793 ; Quelques idées sur la disposition, l'arrangement et la décoration du Muséum national, Paris, impr. de Didot jeune, 1795.

12. Idem, Quelques idées sur la disposition, l'arrangement et la décoration du Muséum national, Paris, impr. de Didot jeune, 1795, p. 26.

13. Louis-Georges de Bréquigny, président pour la création de dépôts, Mémoire, $2^{\mathrm{e}}$ partie, présenté en 1790 à l'Assemblée législative.

14. Dès la Révolution, les administrations successives emploient les termes de " progrès » et de "régénération » des arts dans le cadre de la restructuration des institutions, et dans l'espoir de voir une révolution des arts s'opérer, au même titre que la Nation nouvellement régénérée. Une véritable théorisation autour de cette question voit le jour, demeurant centrale dans les premières décennies du $\mathrm{XIX}^{\mathrm{e}}$ siècle. Voir l'article d'Annie Jourdan: "Politique artistique et Révolution française (1789-1800) : la régénération des arts, un échec ? ", Annales historiques de la Révolution française, vol. 309, $\mathrm{n}^{\circ}$ 1, 1997, pp. 401-421.

15. Le décret du 16 décembre 1792 annonce une répartition des biens saisis entre le Muséum national et les musées qui «pourront être créés dans les départements "; le décret du 10 septembre 1793 reconnaît que les œuvres «de première classe » doivent " être réservées au grand et unique Muséum national ». Voir Chantal Georgel, «L'État et "ses" musées de province », Le Mouvement social, 1992/3 (160) (juil-sept 1992), pp. 65-78, p. 67.

16. Pierre Bénézech, ministre de l'Intérieur, Lettre à l'administration du Musée central des arts en l'an V (1797), citée par Philippe de Chennevières, «Les musées de province », La Gazette des beauxarts, $1^{\mathrm{er}}$ février 1865, vol. XVIII, nº 104, pp. 118-131, p. 119.

17. Louis Tuetey, Procès-verbaux de la commission des Monuments: 1790-1794, 2 vols, Paris, N. Charavay, 1902-1903, cité par Édouard Pommier: «Naissance des musées de province », dans Pierre Nora (dir.), Les lieux de mémoire. t. II, La Nation, Paris, Gallimard, 1997 [1986], p. 1475.

18. F. Rucker, Les origines de la conservation des monuments historiques en France (1790-1830), Paris, Jouve \& Cie, 1913.

19. Édouard Pommier : « Naissance des musées de province », op. cit. note 17, p. 1499.

20. $1^{\mathrm{er}}$ septembre 1801 .

21. Les villes concernées par l'arrêté sont Bordeaux, Caen, Dijon, Lille, Lyon, Marseille, Nancy, Nantes, Rennes, Rouen, Strasbourg, Toulouse, Genève, Bruxelles et Mayence.

22. Édouard Pommier, " Naissance des musées de province ", op. cit. note 17.

23. Idem, ibidem, p. 1471.

24. Dominique Poulot, op. cit. note 10, p. 1.

25. Cat d'exp. Besançon: le plus ancien musée de France, sous la direction de Marie-Lucie Cornillot, Lucien Lerat et. al., Paris, musée des Arts décoratifs, février-avril 1957, Paris, Union centrale des arts décoratifs, 1957, p. XIII.

26. Arles, La Rochelle et Nîmes, voir Édouard Pommier, « Naissance des musées de province », op. cit. note 17, pp. 1473-1474.

27. Rappelons que la loi de vendémiaire An IV organisant l'instruction publique avait évoqué la création de musées dans les départements, uniquement en annexes aux écoles spéciales de dessin, peinture et architecture.

28. Archives du musée des monuments français, 3 vols, Paris, E. Plon, Nourrit et Cie, 1883-1897, tome II, pièce CCII, cité par Dominique Poulot, op. cit. note 10, p. 320.

29. Édouard Pommier, « Naissance des musées de province », op. cit. note 17, p. 1485.

30. Henri Baderou, «Un échange d'œuvres d'art entre les musées de Paris et de province sous la Révolution », Bulletin de la Société de l'histoire de l'art français, 1935, 2, pp. 168-202, p. 170. 
31. Pour une histoire des dons et legs aux musées de province, voir Chantal Georgel, « De l'art et des manières d'enrichir les collections", dans cat. d'exp. La Jeunesse des musées. Les musées de France au XIX ${ }^{e}$ siècle, sous la direction de Chantal Georgel, Paris, musée d'Orsay, 8 février-7 mai 1994, Paris, éditions de la Réunion des musées nationaux, 1994, pp. 232-240.

32. Pour une histoire approfondie de la genèse des collections et de l'aménagement du musée de Dijon, voir cat. d'exp. L'art des collections, bicentenaire du musée des beaux-arts de Dijon: du siècle des Lumières à l'aube d'un nouveau millénaire, sous la direction de Sophie Jugie et Emmanuel Starcky, Dijon, musée des Beaux-Arts de Dijon, 16 juin-9 octobre 2000, Dijon, musée des Beaux-Arts de Dijon 2000.

33. Loïc Vadelorge, op. cit. note 1, p. 12.

34. Dominique Poulot, op. cit. note 10, p. 324.

35. Henri Baderou, « Un échange d'œuvres d'art entre les musées de Paris et de province sous la Révolution ", Bulletin de la Société de l'histoire de l'art français, 1935, 2, pp. 168-202, pp. 169-170.

36. Catalogue des objets contenus dans la galerie du Muséum français, décrété par la Convention nationale le 27 juillet 1793, l'an second de la République française, s. l. [Paris], impr. de C.-F. Patris, 1793, p. 3.

37. Dominique Poulot, Une histoire des musées de France, XVIII $-\mathrm{XX}{ }^{e}$ siècles, Paris, La Découverte, 2005, p. 48.

38. Le chantier est dirigé par Charles-Joseph Le Jolivet, architecte bourguignon.

39. Édouard Pommier, « Naissance des musées de province », op. cit. note 17, p. 1464.

40. Voir note 15.

41. Bruno Foucart, «Le musée du XIX ${ }^{e}$ siècle : temple, palais, basilique », dans Chantal Georgel, La Jeunesse des musées, op. cit. note 31, p. 123.

42. Procès-verbaux de la Commission des monuments, 2 décembre 1790, cités par Dominique Poulot, «Le patrimoine des musées : pour l'histoire d'une rhétorique révolutionnaire ", Genèses vol. 11, 1993, pp. 25-49, p. 39.

43. Ludovic Vitet, Rapport à M. le ministre de l'Intérieur, sur les monuments, les bibliothèques, les archives et les musées des départements de l'Oise, de l'Aisne, de la Marne, du Nord et du Pas-de-Calais, Paris, Impr. royale, 1831.

44. Archives nationales $\mathrm{F} / 21 / 4500$, musées de province.

45. Ibidem.

46. Dominique Poulot, «Le $\mathrm{XIX}^{\mathrm{e}}$ siècle ou le triomphe du musée [compte-rendu]», Annales. Économies, Sociétés, Civilisations, 1993, vol. 48, nº 6, pp. 1648-1657, p. 1654.

47. Amédée Boinet, Le musée d'Amiens, musée de Picardie, peintures, Paris, Laurens, 1928, pp. 4-7.

48. Bruno Foucart, « Le musée du XIX ${ }^{e}$ siècle : temple, palais, basilique », dans Chantal Georgel, La Jeunesse des musées, op. cit. note 31, p. 128.

\section{RÉSUMÉS}

Le Louvre, musée centralisateur à vocation universelle et encyclopédique, constituerait au premier regard un modèle idéal et légitime pour les musées florissants, tout particulièrement lors d'une période telle que la Révolution. Les liens complexes qu'entretiennent les musées de province avec l'administration centrale et le Louvre sont à l'image d'une histoire plurielle de ces institutions, nous apprenant que, façonnés par une histoire profondément locale faite d'initiatives aussi bien personnelles que collectives, les musées connaissent des trajectoires 
multiples, modelées par la prégnance d'enjeux identitaires. C'est entre théorisation et réalité matérielle, en passant par des intentions propres à chaque musée, qu'émergent diverses formes d'appropriation entre les musées.

The Louvre, a central museum with a universal and encyclopaedic vocation, would seem to constitute at first glance an ideal and legitimate model for flourishing museums, particularly during a period such as the Revolution. The complex ties between provincial museums and the central administration and the Louvre are in the image of a plural history of these institutions, teaching us that, fashioned by a profoundly local history made of both personal and collective initiatives, the museums experiencing multiple trajectories, modelled by the import of identity issues. It was between theorisation and material reality, via each museum's intentions, that emerged the various forms of appropriation between museums.

\section{INDEX}

Mots-clés : école des Beaux-Arts, Révolution, école de dessin, apprentissage, musée du Louvre, Muséum central, inventaire, catalogue, province, appropriation, identité, Amiens, Dijon, Toulouse, Besançon

Keywords : Revolution, drawing school, fine arts school, apprenticeship, musée du Louvre, Muséum central, inventory, catalogue, province, appropriation, identity, Amiens, Dijon, Toulouse, Besançon

\section{AUTEUR}

\section{CAMILLE DOUTREMÉPUICH}

Doctorante à l'université de Tours sous la direction de France Nerlich, elle est également rattachée au laboratoire InTRu (Interactions, Transferts, Ruptures artistiques et culturels). Après avoir travaillé durant ses années de master (sous la dir. de Barthélémy Jobert et Thierry Laugée, université Paris IV) sur le peintre dijonnais Anatole Devosge (1770-1850), fils du fondateur de l'École gratuite de dessin de Dijon, elle consacre désormais ses recherches à l'histoire de l'enseignement artistique en province dans la première moitié du XIX ${ }^{\mathrm{e}}$ siècle, à travers la constitution des écoles de dessin et autres institutions en lien avec la formation des artistes et les politiques d'instruction publique (musées, sociétés des amis, ateliers privés).

***

A PhD student at the Université de Tours under the supervision of France Nerlich, she is also associated with the InTRu (Interactions, Transferts, Ruptures artistiques et culturels) laboratory. After working on the Dijon painter Anatole Devosge (1770-1850), son of the founder of the École gratuite de dessin de Dijon, during her master's studies (supervised by Barthélémy Jobert and Thierry Laugée, Université Paris IV), she is now focussing on the history of artistic education in the French provinces in the first half of the nineteenth century, through the constitution of schools of drawing and other institutions associated with the training of artists and the policies of public instructions (museums, friendly societies, private studios). 